\title{
CitRWPMay not be the Major or Only Major Gene that Regulates Apomixis in Citrus and Its Close Related Genus
}

\author{
Yanhong Hou \\ Southwest University \\ Guizhi Gong \\ Southwest University \\ Zhuchun Peng \\ Southwest University \\ Ai Luo \\ Southwest University \\ Yang Cheng \\ Southwest University \\ Qinbin Hong ( $\square$ Hongqibin@cric.cn )
}

\section{Research article}

Keywords: Citrus, Apomixis, Nucellar embryony, CitRWP, cDNA clone

Posted Date: July 27th, 2020

DOI: https://doi.org/10.21203/rs.3.rs-41831/v1

License: (a) (i) This work is licensed under a Creative Commons Attribution 4.0 International License. Read Full License 


\section{Abstract}

Background: Nucellar embryony in citrus and its close related genus is a kind of apomixis, it interferes the cross breeding and affects the genetic evolution and taxonomy in citrus. Many hypotheses have been suggested on regulation of apomixes in citrus but none of them are able to lock the true regulatory sequence until the recently reported candidate gene citRWP. However, the support evidences for the judgment of citRWPas the major or only major gene are still not very sound.

Results: Firstly, M/P (Monoembryonic / Polyembryonic) marker, which was designed according to the upper stream sequence of citRWP, was used to check whether the marker exhibiting consistent segregation in different embryonic genotypes. The result showed that all sweet orange selections either monoembryonic or polyembryonic contained the P-marker, but no P-marker was detected in polyembryonic Poncirus, and a new band pattern was dectected in 9 polyembryonic progenies of W-Murcott $\times$ Flying dragon trifoliate orange. Subsequently, CitRWP expressions were analyzed with RT-PCR and Realtime RT-PCR. RT-PCR revealed that CitRWP expressed in ovule of all varieties. Realtime RT-PCR results in ovule were consistent with RT-PCR. The expression value of monoembryonic pummelo was the lowest, and there was no significant difference between polyembroynic and monoembryonic sweet orange cultivars. In the hybrid offsprings, the expression of the CitRWP gene showed an extreme two-stage differentiation. The expression in young leaf and ovule of polyembryonic and monoembryonic sweet orange cultivars were different, with a higher expression level in young leaf and a lower expression level in ovule of polyembryonic cultivars. Finally, the cDNA sequences of CitRWP from ovules were cloned and sequenced. Results revealed that the CitRWP has great variations in Poncirus, which included SNPs in start-codon and/or stop-codon as well as fragment insertion/deletion causing incorrect translation. The monoembryonic/polyembryonic sweet orange cultivars and pummelo varieties had similar variations also.

Conclusion: CitRWP were not consistently distributed and expressed in different embryonic genotypes. It may not be the major or only major gene that regulates apomixis in citrus and its close related genus.

\section{Background}

Citrus and its close related genus has rich germplasm resources. They originated and distributed in a barrel area from East Asia to Australia [21]. Citrus is the most planted fruit trees in the world, and as its close related genus, Poncirus and its hybrids are the most widely used rootstock. A majority of the cultivated citrus are obtained from natural resource exploration and bud sport selection $[3,22]$. Because many varieties, such as sweet orange, grapefruit and lemon, produce polyembryonic seeds, usually with one zygote embryo and many nucellar embryos or only nucellar embryos within a seed, it is difficult to get hybrid seedlings when these varieties were used as the female parent. Thus, the nucellar embryony interference has become an important factor that hinders systematic and targeted cross-breeding of citrus [9]. Nucellar embryony is a kind of apomixes. According to the origin of embryo, apomixis can be divided into two types, one is gametophytic apomixis whose embryo formed by undiminished embryo sac, and the other is sporophytic apomixis whose embryos formed directly by somatic cells. The citrus nucellar embryony belongs to the latter [12]. Many current research progress reported on apomixis is mainly focused on gametophyte of apomixes [2], and genes controlled the production of unreduced gametes have been discovered [4]. It has been reported that three key genes controlled the meiosis process and apomictic seeds have been successfully obtained in rice by mutation of these genes together to construct an artificially MIME mutant (Mitosis instead of Meiosis) [10,23]. Compared with gametophyte apomixes created by MIME, sporophytic apomixes, represented by citrus, has significantly different genesis and genetic mechanisms, and its formation is 
relatively simpler in terms of materials acquisition, research basis and genetic control process [7]. In the process of transferring apomixis traits to crops, sporophytic apomixis might provide a new supplementary approach for those species to obtain apomixis difficultly by construction of MIME.

Citrus nucellar embryony starts by invasion of nucellar tissues into the embryo sac, then the fertilization and endosperm development further stimulate nucellar embryos, and endosperm provides nutrients for later growth of somatic embryos $[11,25]$. Citrus nucellar embryony shows a simple segregation ratio in the segregating populations, which conforms to the G.J. Mendel's classic genetic law of qualitative traits. There are many theoretical hypotheses supposed to explain the regulation of citrus nucellar embryos, such as single gene [8], single major gene with minor multi-genes [18], and double genes [6]. Contradictory results were found for some hypotheses. For single gene hypothesis, according to Mendel's Law of segregation, only polyembryonic offsprings or half polyembryonic Vs. half monoembryonic offsprings could be found in test cross combination of monoembryonic and polyembryonic genotypes, but different segregation ratios were observed in different hybridization populations. And above all, there is still a gap from hypothesis to manipulatable gene sequences.

In recent years, progresses have been made from hypothesis to manipulatable gene sequences. Garcia et al found six QTLs that control apomixis in citrus [5], and Kepiro and Roose reported five candidate AFLP markers that are closely linked to the polyembryonic Poncirus [13]. Nakano et al obtained SNP and CAPS markers co-segregated with polyembryonic varieties by screening in the flanking region of polyembryonic sites, and used these markers to lock a polyembryony control region which contained $380 \mathrm{kbp}$ sequence with 70 predicted open reading frames (ORFs) [16, 17]. Shimada et al further tested this region [20]. They designed DNA microarray to check the gene expression of all ORFs in the polyembryony control region, and a candidate gene CitRKD1 was screened and tested by RNAi silencing with a polyembryonic sweet orange variety. Meanwhile, an M/P marker was designed for the presence / absence of MITE insertion in the promoter region of CitRKD1. Similar result was reported by Wang et al [24] and they narrowed the polyembryony control region to $80 \mathrm{kbp}$, which contains 11 candidate genes. After analyzing the expression of these genes, they conferred that CitRWP with MITE insertion in the promoter region was a key candidate gene for controlling citrus somatic embryogenesis. As the sequence of CitRWP and CitRKD1 was the same, this gene was referred as CitRWP hereafter.

However, the support evidences for the judgment of CitRWP as the major or only major gene is still not very sound. CitRWP encodes a RWP-RK domain-containing protein similar to the Arabidopsis RKD family of proteins [24] and this protein is found to serve as regulators of egg cell-related genes in Arabidopsis [15]. But no nucellar embryony or polyembryony was reported in Arabidopsis so far. The gene expression was found to be co-related with nucellar embryony in citrus materials with very similar genetic background and whether this co-relation could be found in genetically diversified genotypes? It is important for a gene to be considered a target in genetic modification in a new genetic background such as cereal crops. Relative genus of citrus, where very similar apomixis genes and regulating mechanism might exist, is a good choice to test the gene expressions. Shimada et al knocked out CitRWP by antisense-overexpression in a polyembryonic sweet orange, but only one genetic modified plant showed monoembryonic trait [20]. Somatic mutation is common in citrus, especially under stress conditions as tissue culture. So it is difficult to judge that the monoembryonic change is the result of gene knock-out of CitRWP or the mutations of other genes.

For the sake of successful and effective harnessing of CitRWP in other crops, there are still needs to further evaluate the gene function of citRWP. We carried out this work with different embryonic genotypes of very similar genetic 
background or far diversified genetic background from three aspects: M/P marker check in genome and relative expression detection as well as cDNA cloning and sequencing.

\section{Results:}

\section{Validation of M/P markers in different embryonic genotypes}

The M/P marker was designed by Shimada et al [20]. M-marker implied to monoembryonic alleles with $0.75 \mathrm{~kb}$ fragments and P-marker implied to polyembroynic alleles with $1.0 \mathrm{~kb}$ fragments. By the genotyping analysis of 95 traditional and breeding varieties, they found that P-marker could be detected in all polyembryonic varieties and only M-marker could be detected in monoembryonic varieties. However, inconsistent results were observed in our study.

Firstly, P marker were detected in three monoembryonic sweet orange varieties (Li2, TD, MS) and their M/P patterns were the same as two polyembryonic sweet orange varieties (Tarocco blood orange and Hamlin sweet orange). Only M-marker were detected in monoembryonic Banpeiyu pummelo and clementine mandarin (Fig. 1). Sweet orange is the most planted citrus variety and most sweet orange varieties produce polyembryonic seeds, but monoembryonic lines were occasionally found around the world also. Our monoembryonic sweet orange varieties were found during our breeding process and citrus germplasm collection and evaluation in China. They should be somatic mutation origin. According to the results, the CitRWP of monoembryonic sweet orange should have the MITE insertions also. It is contradictory with conclusion "polyembryonic alleles with a MITE insertion in the upstream region and monoembryonic alleles without it" proposed by Shimada et al [20].

Subsequently, only M-marker was detected in different Poncirus genotypes, a close related genus of Citrus (Fig. 2). A reasonable deduction might be that the CitRWP exists in Poncirus and the gene does not contain the MITE insertion or a huge mutation occurred in upstream. The marker gave a different scenario in offsprings of W-Murcott $\times$ Flying dragon trifoliate orange (Fig. 2). Only M-marker was detected in all 7 progenies, P-marker together with Mmarker were detected in 9 progenies. But a different band pattern was found in both P-marker and M-marker progenies. 4 polyembryonic genotype offsprings produced the same band pattern as the seed parent W-Murcott with a stronger M-marker band and a weaker P-marker band, and 3 polyembryonic progenies produced new band pattern. The new band pattern included a stronger and longer band than the P-marker band and M-marker band.

\section{CitRWP expression in different materials}

Nucellar embryo initiates and develops in the ovule, and the flowering stage is the critical period for the development of nucellar embryo, so gene expression analysis was done in ovules of different embryogenic varieties at flowering stage. CitRWP expression was detected by RT-PCR with Actin as the reference gene. Under same mRNA extraction and reverse transcription procedure and PCR conditions, RT-PCR revealed that CitRWP expressed in all varieties (Fig. 3). Judged by the strength of band, the expression level of monoembryonic Longan pummelo was the lowest, followed by polyembryonic Flying dragon trifoliate orange and Ponkan, and all the remaining samples showed very similar expression strength. No obvious difference could be discriminated between monoembryonic sweet orange varieties and polyembryonic sweet orange varieties.

CitRWP expression in ovule was further checked by qRT-PCR in two consecutive years. REV (Relative expression values) of different embryonic varieties were computed with Banpeiyu pummelo as control. The REV of Ponkan was similar with polyembryonic sweet orange Hamlin and Tarocco blood orange, while the expression value of WMurcott was similar to those of monoembryonic sweet orange (Fig. 4). In the 2019 CitRWP expression test, the REV 
of Flying dragon trifoliate orange was 5.5 times that of monoembryonic Banpeiyu pummelo, and the REV of polyembryonic sweet orange Hamlin and Tarocco blood orange was 73.1 and 46.9 times that of Banpeiyu pummelo, respectively. The REV of monoembryonic sweet orange Licheng No. 2, TD and MS was 21.7 times, 32.14 times, and 42.9 times that of Banpeiyu pummelo, respectively. Notably, the REV of CitRWP in the polyembryonic Flying dragon trifoliate orange was much lower than that of the monoembryonic sweet orange, and slightly higher than that of the monoembryonic Banpeiyu pummelo, such as the REV of monoembryonic sweet orange MS was 7.7 times that of polyembryonic Flying dragon trifoliate orange. In addition, The CitRWP expression value was not significantly different between monoembryonic and polyembryonic sweet oranges. REV of Hamlin (the highest in polyembryonic sweet orange) was 3.36 times that of Licheng No.2 (the lowest REV in monoembryonic sweet oranges), the REV of Tarocco blood orange was only 1.1 times that of monoembryonic MS sweet orange (Fig. 4). These results showed that the expression of CitRWP might not directly related to embryonic phenotypes.

The ovule in citrus and its close relative genus is very tiny during flowering time and young fruit stage. It is difficult to get enough pure ovule for experiment quickly and safely. So we carried out a correlation analysis on the expression of ovule and ovary at the same period. The result showed that the CitRWP expression of ovary was correlated with the ovule positively significantly (Fig. 6). Therefore, the expression in ovary was detected in the offspring population of W-Murcott $\times$ Flying dragon trifoliate orange. The analysis found that the CitRWP REV in WMurcott was 6.43 times that of Flying dragon trifoliate orange, and the REV of Wangcang daye trifoliate orange and 74 - 1 trifoliate orange were similar to that of Flying dragon trifoliate orange. In the hybrid offspring population, the REV of CitRWP gene was an extreme two-stage differentiation, there were 5 high-expressing progenies, 4 progenies of which were higher than their female parent W-Murcott, while 9 low-expressing progenies lower than their male parent Flying dragon trifoliate orange. The REV of high-expressing progenies was ten or even hundreds of times that of low-expression. Among the tested progenies, the high-expressing lines had been recorded as polyembryony in the embryony investigation, and the low-expression lines was monoembryonic, which indicated that the REV of CitRWP might have correlation with embryonic phenotype in the W-Murcott $\times$ Flying dragon trifoliate orange hybrid offspring population (Fig. 7).

In addition to the test of CitRWP expression in ovule, we also tested the expression of different tissues or organs such as young stem, young leaf, old leaf, and ovary of mono/polyembryony sweet orange. Among the polyembryonic Hamlin and Tarocco blood orange, the young leaf had the highest expression value which was slightly lower than its ovule. However, the expression value of leaf decreased significantly after maturity. REV in young leaves of Hamlin and Tarocco blood orange was 2.5, 3.2 times that of its old leaves, respectively. Among the monoembryonic sweet orange, the ovule was the part with highest expression, and the young leaf expression value had no significant difference with the young stem, old leaf, and ovary (Fig. 8). CitRWP expressed in vegetative tissue, especially the young leaf in polyembryonic orange had the highest REV, indicating that CitRWP was not specifically expressed in reproductive tissue.

\section{Cloning and sequencing of citRWP from different embryonic materials}

cDNAs of CitRWP were cloned and sequenced from ovules of 3 polyembryonic Poncirus cultivars, 2 mono/polyembryonic sweet orange selections and 2 monoembryonic pummelos. CDS sequence of Cs4g05960 in sequenced sweet orange [25] was used as standard to compare with the cloned citRWP sequences. Three types of major variations were observed in the obtained sequences of polyembryonic Poncirus (Fig. 9). These variations appeared in almost all Poncirus cultivars with a high rate (Table. 1). The type I variation was the deletion of a $106 \mathrm{bp}$ fragment at the $406 \mathrm{bp}$ position (relative to the translation start codon, the same hereinafter) and this 
deletion caused the CDS length to change from $1065 \mathrm{bp}$ to $959 \mathrm{bp}$. The primers used for RT-PCR were found to locate in this region, so it might be one of the reason that caused a lower REV in polyembryonic Poncirus. 8 in 18 clones presented the $106 \mathrm{bp}$ fragment deletion mutation. The type II variation was a 5-base (-TGCAG-) insertion mutation at the 774 bp position, which caused the CDS length changed from 1065 bp to 1070 bp. This variation presented in 8 sequenced clones. Two types of the variations coexisted in 2 sequenced clones and the CDS length of these sequences was $964 \mathrm{bp}$. The length of these three CDS sequences was not integral multiple of triplet codons, so they could not be translated normally. The type III variation was mutations in initiation codon and termination codon. The key codon changed also lead to mistranslation of functional protein. In general, the 18 sequenced samples of Poncirus, only 3 sequenced clones could be normally translated, and the normal translation rate was only $16.7 \%$.

Less variations were found in cloned citRWP cDNA sequences from 2 sweet orange cultivars and 2 pummelo cultivars. The type I variation of a 106-base deletion was found only in one sequenced clone from Banpeiyu pummelo. The type II variation of a 5-base (-TGCAG-) insertion was found only in four sequenced clones, one from polyembryonic Tarocco blood orange and three from monoembryonic sweet orange TD. No type III variation in initiation codon and termination codon was found. The overall normal translation rate was $69.6 \%$ in sweet orange and pummelo, which was higher than that in Poncirus. In addition, compared with Cs4g05960, there are 5 SNP sites in the sequenced pummelo clone, but they are all synonymous mutations and do not change the encoded amino acids.

\section{Discussion:}

Nucellar embryony is widely considered a qualitative trait and many theoretical hypotheses were supposed to explain the regulation of citrus nucellar embryony $[6,8,18]$. The single gene hypothesis could explain only a part of the embryony investigation results. Hypothesis of two complementary dominant genes controlling apomixis in genus citrus and poncirus gave a better explanation [6]. The hypothesis could explain genotypes controlling polyembryony / monoembryony existing both in natural species and artificial hybridization progenies as well as most of the known hybridization results. According to this hypothesis, it is difficult to screen both control genes at the same time with a generalized hybrid segregation population by BSA method (Bulk Segregant Analysis) or methods alike. Furthermore, the genes in classic genetics are not corresponded to mRNA encoding sequences one for one and they might include regulatory sequences as miRNAs or circRNAs. The expression analyses of mRNA in a specified sequence area might confine the exploration of more possibilities.

CitRWP gene was considered as the candidate apomixis gene by two different research groups recently $[19,24]$. BSA method and following gene expression analyses were key steps in their screening work. By BSA with RAPD markers and mapping on a linkage map population, a polyembryony locus was detected. The draft sequence corresponded to polyembryony locus were then obtained through markers enrichment [16] and haplotype-specific physical maps construction [17]. DNA microarray were designed to check the gene expression of all ORFs in the polyembryony control region, and CitRWP was confirmed as the candidate gene [20]. The confirmation of CitRWP with MITE insertion as a key candidate polyembryony gene by Wang et al were in a similar way [24]. Firstly, a polyembryony related region spanning $1.96 \mathrm{Mb}$ on chromosome 4 was found by BSA in a segregating population of HB pummelo and Fairchild mandarin. Subsequently, the region was narrowed to $\sim 80-\mathrm{kb}$ by a local association analysis with the variant information from the protein-encoding genes using the genome sequencing data from 108 accessions. Finally, the expression analysis of all candidate genes in the $\sim 80-\mathrm{kb}$ region helped to lock CitRWP. 
CitRWP contains the conserved RWP-RK domain. Genes with this domain are widely distributed in plants [19]. They can be divided into two subfamilies, the NLP subfamily and the RKD subfamily. The former is related to nitrogen fixation, and the latter is related to plant reproduction and widely distributed in lower plants to higher plants [14] and is involved in egg cell specification and differentiation [1]. CitRWP belongs to the later and was named as CitRKD1 also [20]. However, only a few species show the nucellar embryony phenomenon, which may lead to the doubt whether CitRWP is a major gene in citrus nucellar embryony regulation.

In this work, CitRWP was studied from three aspects and converged data did not give necessary and sufficient support for its role in regulating nucellar embryony in citrus, let alone Poncirus. There were contradictions in facts as P-markers detected in monoembryonic sweet orange and no significantly different CitRWP expression value between monoembryonic and polyembryonic sweet oranges, as well as identical mRNA sequences among monoembryonic and polyembryonic sweet oranges. Similar contradictions existed in polyembryonic Poncirus also. CitRWP did not get full support as a candidate gene for nucellar embryony regulation in the offsprings of apomictic tangerine $\times$ apomictic trifoliate orange also [25].

Genes regulating nucellar embryony have their values in cross breeding and genetic evolution and taxonomy research in citrus. More important and valuable application of these genes might lies in the transferring of apomixis traits to other crops. Whether genes function desirable in citrus could exhibit the same function in a far different genetic background, more intensive studies are needed still.

\section{Conclusions}

CitRWP were not consistently distributed and expressed in different embryonic genotypes. CitRWP may not be the major or only major gene that regulates apomixes in citrus and its close related genus. The step to approach the regulating mechanism of citrus apomixes is still on the way.

\section{Method}

\section{Plant materials}

Different embryonic materials

All testing materials in this experiment were planted in the Citrus Research Institute, Southwest University, Chongqing, China. The type of embryony is shown in Table 2. 
Table 1

variations in citRWP CDNA clones

\begin{tabular}{|c|c|c|c|c|c|c|c|c|}
\hline $\begin{array}{l}\text { Scientific } \\
\text { name }\end{array}$ & $\begin{array}{l}\text { Common } \\
\text { name }\end{array}$ & $\begin{array}{l}106 \text { bp } \\
\text { deletion } \\
\text { at } \\
406 \text { bp }\end{array}$ & $\begin{array}{l}5 \text { bp } \\
\text { insert } \\
\text { at } \\
774 \text { bp }\end{array}$ & $\begin{array}{l}\text { Initiation or } \\
\text { termination } \\
\text { codon } \\
\text { change }\end{array}$ & $\begin{array}{l}\text { Number of } \\
\text { normal } \\
\text { translation } \\
\text { clones }\end{array}$ & $\begin{array}{l}\text { Number } \\
\text { of Clones } \\
\text { with two } \\
\text { or more } \\
\text { mutations }\end{array}$ & $\begin{array}{l}\text { Total } \\
\text { clones }\end{array}$ & $\begin{array}{l}\text { Proportion } \\
\text { of normal } \\
\text { translation }\end{array}$ \\
\hline \multirow[t]{3}{*}{$\begin{array}{l}\text { Poncirus } \\
\text { trifoliata } \\
\text { (L.) Raf }\end{array}$} & $\begin{array}{l}\text { Flying } \\
\text { dragon } \\
\text { trifoliate } \\
\text { orange }\end{array}$ & 4 & 4 & 1 & 1 & 2 & 8 & $12.5 \%$ \\
\hline & $\begin{array}{l}\text { Wangcang } \\
\text { daye } \\
\text { trifoliate } \\
\text { orange }\end{array}$ & 2 & 1 & 0 & 2 & 1 & 5 & $20 \%$ \\
\hline & $\begin{array}{l}74-1 \\
\text { trifoliate } \\
\text { orange }\end{array}$ & 2 & 3 & 2 & 1 & 1 & 5 & $20 \%$ \\
\hline \multirow[t]{2}{*}{$\begin{array}{l}\text { Citrus } \\
\text { sinensis } \\
\text { (L.) } \\
\text { Osbeck }\end{array}$} & $\begin{array}{l}\text { Tarocco } \\
\text { blood } \\
\text { orange }\end{array}$ & 0 & 1 & 0 & 4 & 0 & 5 & $80 \%$ \\
\hline & $\begin{array}{l}\text { Tiandan } \\
\text { sweet } \\
\text { organe }\end{array}$ & 0 & 3 & 0 & 5 & 0 & 8 & $62.5 \%$ \\
\hline \multirow{2}{*}{$\begin{array}{l}\text { Citrus } \\
\text { maxima } \\
\text { (Burm) } \\
\text { Merr }\end{array}$} & $\begin{array}{l}\text { Longan } \\
\text { pummelo }\end{array}$ & 0 & 0 & 0 & 3 & 0 & 5 & $100 \%$ \\
\hline & $\begin{array}{l}\text { Wanbai } \\
\text { pummelo }\end{array}$ & 1 & 0 & 0 & 4 & 0 & 5 & $80 \%$ \\
\hline
\end{tabular}


Table 2

Embryony type of tested materials

\begin{tabular}{|lll|}
\hline Common name & Scientific name & Embryony type \\
\hline Meiwa kumquat & Fortunella. rassifolia & Polyembryonic \\
Wangcang daye trifoliate orange & Poncitrus. trifoliate & Polyembryonic \\
Flying dragon trifoliate orange 1 trifoliate orange & Poncitrus. trifoliata & Polyembryonic \\
Tarocco blood orange & Citrus sinensis (L.) Osbeck & Polyembryonic \\
Hamlin sweet orange & Citrus sinensis (L.) Osbeck & Polyembryonic \\
Licheng No. 2 sweet orange & Citrus sinensis (L.) Osbeck & Polyembryonic \\
Masai sweet orange & Citrus sinensis (L.) Osbeck & Monoembryonic \\
Tiandan sweet orange & Citrus sinensis (L.) Osbeck & Monoembryonic \\
Daidai sour orange & Citrus aurantium (L.) cV. amara & Monoembryonic \\
W-Murcott tangor & Citrus reticulata Blanco cv. W-Murcott & Polyembryonic \\
Ponkan & Citrus reticulata Blanco & Polyembryonic \\
Clementina & Citrus clementina Hort & Monoembryonic \\
Longan pummelo & Citrus grandis Osbeck & Monoembryonic \\
Banpeiyu pummelo & Citrus grandis Osbeck & Monoembryonic \\
\hline
\end{tabular}

Ovules preparation. Ovaries at blossom time or $1 \sim 2$ DAF were collected. They were cut into halves and ovules were stripped out as clean as possible and put immediately in liquid nitrogen, and stored at $-80^{\circ} \mathrm{C}$ for RNA isolation.

DNA preparation. Young leaves were used to extract total DNA with CTAB method. After DNA was precipitated by isopropanol and dissolved in water, RNaseA was added at a final concentration of $20 \mu \mathrm{g} \cdot \mathrm{mL}^{-1}$ and incubated at $37^{\circ} \mathrm{C}$ for 1 hour. DNA concentration and quality were measured with a fluorometer (Biorad, San Francisco, CA, USA) in combination with agarose gel electrophoresis.

RNA extraction. Total RNA was extracted with the EASY spin Plant RNA Rapid Extraction Kit (Biomed, Beijing, China) from prepared plant materials above. RNA integrity was checked with $1.0 \%(\mathrm{~W} / \mathrm{V})$ agarose gel electrophoresis.

cDNA preparation. The first strand cDNA was synthesized from intact RNA using the HiScript II Q RT SuperMix for qPCR (+ gDNA wiper) (vazyme, China) kit according to the manual. The products was stored at $-20^{\circ} \mathrm{C}$ for further use.

\section{M/P marker detection}

The M/P marker primers were synthesized according to Shimada [20]. The primer sequences were 5'tctggttcattgagaatcc-3' and 5'-ctgagcaccaggcaacacacac-3'.

The PCR reaction was performed in $20 \mu$ l volume, which was consisted of about $80 \mathrm{ng}$ of template genomic DNA, $0.2 \mathrm{mM}$ dNTPs, $0.2 \mu \mathrm{M}$ forward and reverse primers, $1 \times$ PCR buffer, $2.0 \mathrm{mM}$ magnesium chloride and 1U Taq DNA 
polymerase. The amplification procedure was as follows: $94^{\circ} \mathrm{C}$ for $4 \mathrm{~min} ; 10$ cycles of $94{ }^{\circ} \mathrm{C}$ for $45 \mathrm{~s}, 64^{\circ} \mathrm{C}$ for $40 \mathrm{~s}$, and $72{ }^{\circ} \mathrm{C}$ for $45 \mathrm{~s}$; and then 23 cycles of $94^{\circ} \mathrm{C}$ for $45 \mathrm{~s}, 58{ }^{\circ} \mathrm{C}$ for $40 \mathrm{~s}$, and $72{ }^{\circ} \mathrm{C}$ for $45 \mathrm{~s}$; and a final cycle of $72{ }^{\circ} \mathrm{C}$ for $5 \mathrm{~min}$. PCR reactions were performed on a Biometra T1 thermocycler (Norwalk, CT, USA). The PCR products were analyzed by $1.5 \%$ (W/V) agarose gel electrophoresis.

\section{RT-PCR and Realtime RT-PCR detection}

RT-PCR reaction was performed in a GeneAmp PCR system (Applied Biosystems, USA). The primers were synthesized according to Zhang Siqi [25]. The primer sequences for CitRWP were 5'-CTGGCGTTCCTCGGTCACT-3' and 5'-TGCAATGCTGGGAGTTTTCA-3'. The sequences for Actin were 5'-CATCCCTCAGCACCTTCC-3' and 5'CCAACCTTAGCACTTCC-3'. The PCR reaction was performed in $20 \mu$ volume, which was consisted of about $100 \mathrm{ng}$ of template cDNA, $0.2 \mathrm{mM}$ dNTPs, $0.2 \mu \mathrm{M}$ forward and reverse primers, $1 \times$ PCR buffer, $2.0 \mathrm{mM}$ magnesium chloride and $1 \mathrm{U}$ Taq DNA polymerase. The amplification procedure was as follows: pre-denaturation 4 min at $94^{\circ} \mathrm{C}$, denaturation $45 \mathrm{~s}$ at $94^{\circ} \mathrm{C}$, annealing temperature $45 \mathrm{~s}$ at $60^{\circ} \mathrm{C}$, and extension $40 \mathrm{~s}$ at $72{ }^{\circ} \mathrm{C}, 40$ cycles; 3 min extension at $72{ }^{\circ} \mathrm{C}$, and stored at $4{ }^{\circ} \mathrm{C}$. The PCR products were analyzed by $1.5 \%(\mathrm{~W} / \mathrm{V})$ agarose gel electrophoresis.

The Realtime RT-PCR was performed in ABI 7500 (Applied Biosystems). The same cDNA template and primers set were used as in RT-PCR. The reaction system is based on the instructions of AceQ ${ }^{\circledR}$ qPCR SYBR Green Master Mix (Vazyme, Nanjing, China). The reaction program is as follows: $5 \mathrm{~min}$ at $95^{\circ} \mathrm{C}$; $10 \mathrm{~s}$ at $95^{\circ} \mathrm{C}, 1 \mathrm{~min}$ at $60{ }^{\circ} \mathrm{C}, 40$ cycles; using $2^{-\Delta \Delta \mathrm{Ct}}$ method to calculate the value of relative expression.

\section{CitRWP cloning and sequencing}

cDNAs from ovules of selected polyembryonic and monoembryonic sweet orange and pummelo cultivars were used to clone CitRWP full-length sequence. The primer sequences for cloning were as follows: CLONE-F: 5'-

ATGGCGGATCCTAGgGCCAT-3', CLONE-R: 5'-ATGTGCTGGACTCACTAGATGA-3'. PrimeSTAR Max DNA polymerase (Takara), a fast and high-fidelity DNA Polymerase was used in PCR amplification. The PCR program is designed as follows: 5 min at $95^{\circ} \mathrm{C}$, then $15 \mathrm{~s}$ at $94^{\circ} \mathrm{C}, 30 \mathrm{~s}$ at $58{ }^{\circ} \mathrm{C}, 1 \mathrm{~min}$ at $72{ }^{\circ} \mathrm{C}, 30$ cycles; $3 \mathrm{~min}$ at $72{ }^{\circ} \mathrm{C}$, and stored at $4{ }^{\circ} \mathrm{C}$. The obtained product was added an A-tail and was transformed into the PGEM-Teasy vector (Promega) by the high efficiency cloning reagent kit pGEM®-T Easy Vector Systems (Promega). The obtained vector was transformed into E.coli DH5a(Takara) and screened by blue-white selection. The successful clones were picked at random and sent to Tsingke biological technology (Beijing, China) for sequencing.

\section{Abbreviations}

AFLP

Amplified fragment length polymorphism; BSA:Bulk segregant analysis; CAPS:Cleaved amplified polymorphism sequences; CDS:Coding sequence; DAF:Days after flowering; MITE:Miniature inverted-repeat transposable element; MIME:Mitosis instead of Meiosis; M/P marker:Monoembryonic / Polyembryonic marker; NLP:NIN-like protein; ORF:Open reading frames; QTL:Quantitative trait locus; RAPD:Random amplified polymorphic DNA; REV:Relative expression values; RKD:RWP-RKD domain-containing; RNAi:RNA interference; SNP:Single nucleotide polymorphism

\section{Declarations}

\section{Ethics approval and consent to participate}

Not applicable. 


\section{Consent to publish}

Not applicable.

\section{Availability of data and materials}

The CitRWP sequence was downloaded from the citrus.hzau database (http://citrus.hzau.edu.cn/cgibin/orange/gene/Cs4g05960.1). Sequence align and analysis on DNA Star. The plant materials are available from the corresponding author upon reasonable request.

\section{Acknowledgements}

Not applicable.

\section{Competing interests}

The authors declare that they have no competing interests.

\section{Funding}

we thank The National Key Research and Development Program of China (2019YFD1001402), providing the financial support for the cloning and sequencing analysis $₫$ The National Key Technology R\&D Program of China (2013BAD02B02) provided us financial support for M/P marker, RT-PCR and Realtime RT-PCR detection.

\section{Authors' contributions}

$\mathrm{H}-\mathrm{YH}$ and Q-BH designed the experiments. $\mathrm{H}-\mathrm{YH}$ conducted the experiments and data analysis and wrote the first draft of the manuscript. Q-BH and G-ZG edited the manuscript. Z-CP, Q-BH and G-ZG constructed the offsprings of W-Murcott $\times$ Flying dragon trifoliate orange. CY and AY helped with ovules preparation and conducted RNA preparation. All authors have read and approved the final manuscript.

\section{Authors' information}

\section{Affiliations}

Citrus Research Institute, Southwest University/Chinese Academy of Agricultural Sciences, Beibei, Chongqing 400712, China

National Citrus Engineering Research Center, Beibei, Chongqing 400712,China

Yanhong Hou, Guizhi Gong, Zhuchun Peng, Ai Luo, Cheng Yang, Qibin Hong

Corresponding author

Correspondence to Qinbin Hong

\section{References}

1. Chardin C, Girin T, Roudier F, Meyer C, Krapp A. The plant RWP-RK transcription factors: key regulators of nitrogen responses and of gametophyte development. J Exp Bot. 2014;65:5577-87. 
2. Conner JA, Mookkan M, Huo H, Chae K, Ozias-Akins P. A parthenogenesis gene of apomict origin elicits embryo formation from unfertilized eggs in a sexual plant. Proc. Natl. Acad. Sci. USA. 2015; 112: 11205-11210.

3. Deng XX. Advances in worldwide citrus breeding. Acta Horticulturae Sinica. 2006;32(6):1140-5.

4. Fei XT, Shi JW, Liu YL, Niu JS, Wei AZ. The steps from sexual reproduction to apomixes. Planta. 2019;249:1715-30.

5. Garcia R, Asins M, Fomer J, Carbonell E. Genetic analysis of apomixis in Citrus and Poncirus by molecular markers. Theor Appl Genet. 1999;99:511-8.

6. Hong QB, Xiang SQ, Chen KL, Chen LG. Two complementary dominant genes controlling apomixis in genus Citrus and Poncirus. Acta Genet Sin. 2001;28:1062-7.

7. Hou YH, Gong GZ, Peng ZC, Dong QQ, Luo Al, Hong QB. MIME-Mitosis instead of Meiosis and its application in crop apomixes. Chin J Biotech, 2020; 612-621.

8. Iwamasa M, Ueno I, Nishiura M. Inheritance of nucellar embryony in Citrus. Bull Hort Res Sta Japan. 1967;7:18.

9. Kepiro JL, Roose ML. Nucellar embryony. In: Citrus. genetics, breeding and biotechnology, editor I.Kan.CABI. 2007; 141-149.

10. Kepiro JL, Roose ML. AFLP markers closely linked to a major gene essential for nucellar embryony (apomixis) in Citrus maxima × Poncirus trifoliate. Tree Genet Genome. 2010;6:1-11.

11. Khanday I, Skinner D, Yang B, Mercier R, Sundaresan V. A male-expressed rice embryogenic trigger redirected for asexual propagation through seeds. Nature. 2019;565:91-5.

12. Koltunow AM, Soltys It, Nito N, McClure S. Anther, ovule, seed, and nucellar embryo development in Citrus sinensis cv. Valencia. Can J Bot. 1995;73:1567-82.

13. Koltunow AM. Apomixis: embryo sacs and embryos formed without meiosis or fertilization in ovules. Plant cell. 1993;5:1425-37.

14. Koi S, Hisanaga T, Sato K, Shimamura M, Yamato KT, Ishizaki K, Kohcki T. Nakajima K.An evolutionarily conserved plant RKD factor controls germ cell differentiation. Curr Biol. 2016;26:1775-81.

15. Köszegi D, Johnston AJ, Rutten T, Czihal A, Altschmied L, Kumlehn J. Members of the RKD transcription factor family induce an egg cell-like gene expression program. Plant J. 2011;67:280-91.

16. Nakano M, Shimizu T, Fuji H, Shimada T, Endo T, Nesumi H, Kuniga T. OmuraM.Marker enrichment and construction of haplotype-specific BAC contigs for the polyembryony genomic region in Citrus. Breeding Sci. 2008;58:375-83.

17. Nakano M, Shimada T, Endo T, Fujii H, Nesumi H, Kita M, Ebina M, Shimizu T, Omura M. Characterization of genomic sequence showing strong association with polyembryony among diverse Citrus species and cultivars, and its synteny with Vitis and Populus. Plant Sci. 2012;183:131-42.

18. Parlevliet JE, Cameron JW. Evidence on the inheritance of nucellar embryony incitrus. Proc Am Soc Hortic Sci. 1959;74:252-60.

19. Schauser L, Wieloch W, Stougaar J. Evolution of NIN-like proteins in Arabidopsis, rice, and Lotus japonicus. J Mol Evol. 2005;60:229-37.

20. Shimada T, Endo T, Fujii H, et al. MITE insertion-dependent expression of CitRKD1 with a RWP-RK domain regulates somatic embryogenesis in citrus nucellar tissues [J]. Bmc Plant Biology. 2018;18:166-85.

21. Swingle WT, Reece PC. The botany of citrus and its wild relatives. In: Reuther W, Webber HJ, Batchelor DL, editors. The Citrus industry. 2nd ed. Vol. 1. Berkley: University of California Press; 1967. pp. 190-422. 
22. Talon M, Gmitter FG. Citrus genomics. Int J Plant Genom. 2008; ID528361.

23. Wang C, Liu Q, Shen Y, et al. Clonal seeds from hybrid rice by simultaneous genome engineering of meiosis and fertilization genes. Nat Biotechnol. 2019;37(3):283-6.

24. Wang X, Xu Y, Zhang S, et al. Genomic analyses of primitive, wild and cultivated citrus provide insights into asexual reproduction. Nat Genet. 2017;49:765-72.

25. Siqi Zhang. Genetic analysis of citrus apomixis and its related genes discovery. Huazhong Agricultural University, Wuhan: PhD diss; 2017.

\section{Figures}

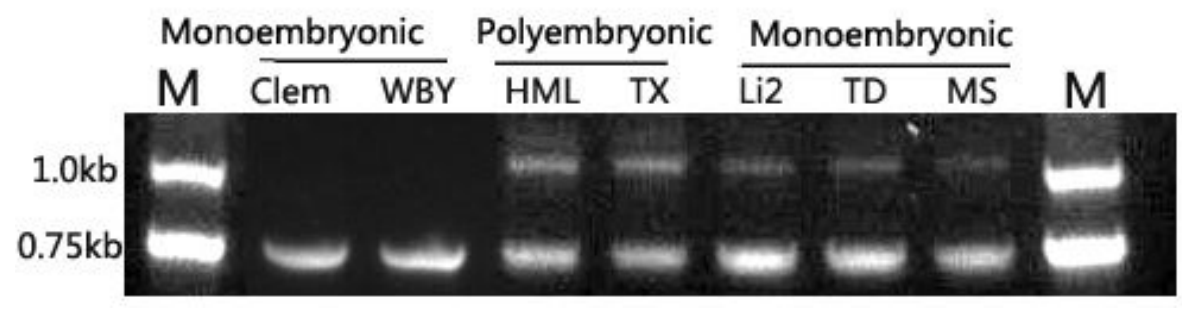

Figure 1

Allelic genotyping of CitRWP alleles in different embryonic sweet orange using M/P marker. The $1.0 \mathrm{kbp}$ fragment represents the polyembryonic allele $(P)$ and the $0.75 \mathrm{~kb}$ fragment represents the monoembryonic allele (M). Clem: Clementina, WBY: Banpeiyu pummelo, HML: Hamlin sweet orange, TX: Tarocco blood orange, Li2: Licheng No.2 sweet orange, TD: Tiandan sweet orange, MS: Masai sweet orange.

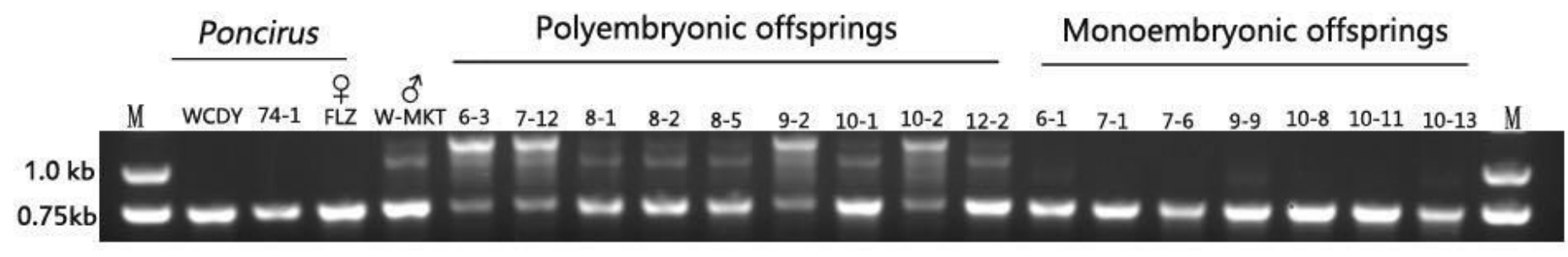

\section{Figure 2}

Allelic genotyping of CitRWP alleles in trifoliate orange genotypes and offsprings of W-Murcott tangor $\times$ Flying dragon trifoliate orange using M/P marker. The $1.0 \mathrm{kbp}$ fragment represents the polyembryonic allele $(P)$ and the $0.75 \mathrm{~kb}$ fragment represents the monoembryonic allele (M). WCDY: Wangcang daye trifoliate orange, 74-1: 74-1 trifoliate orange, FLZ: Flying dragon trifoliate orange, W-MKT: W-Murcott tangor, the remained are offsprings of Flying dragon trifoliate orange $\times$ W-Murcott tangor polyembryonic. 


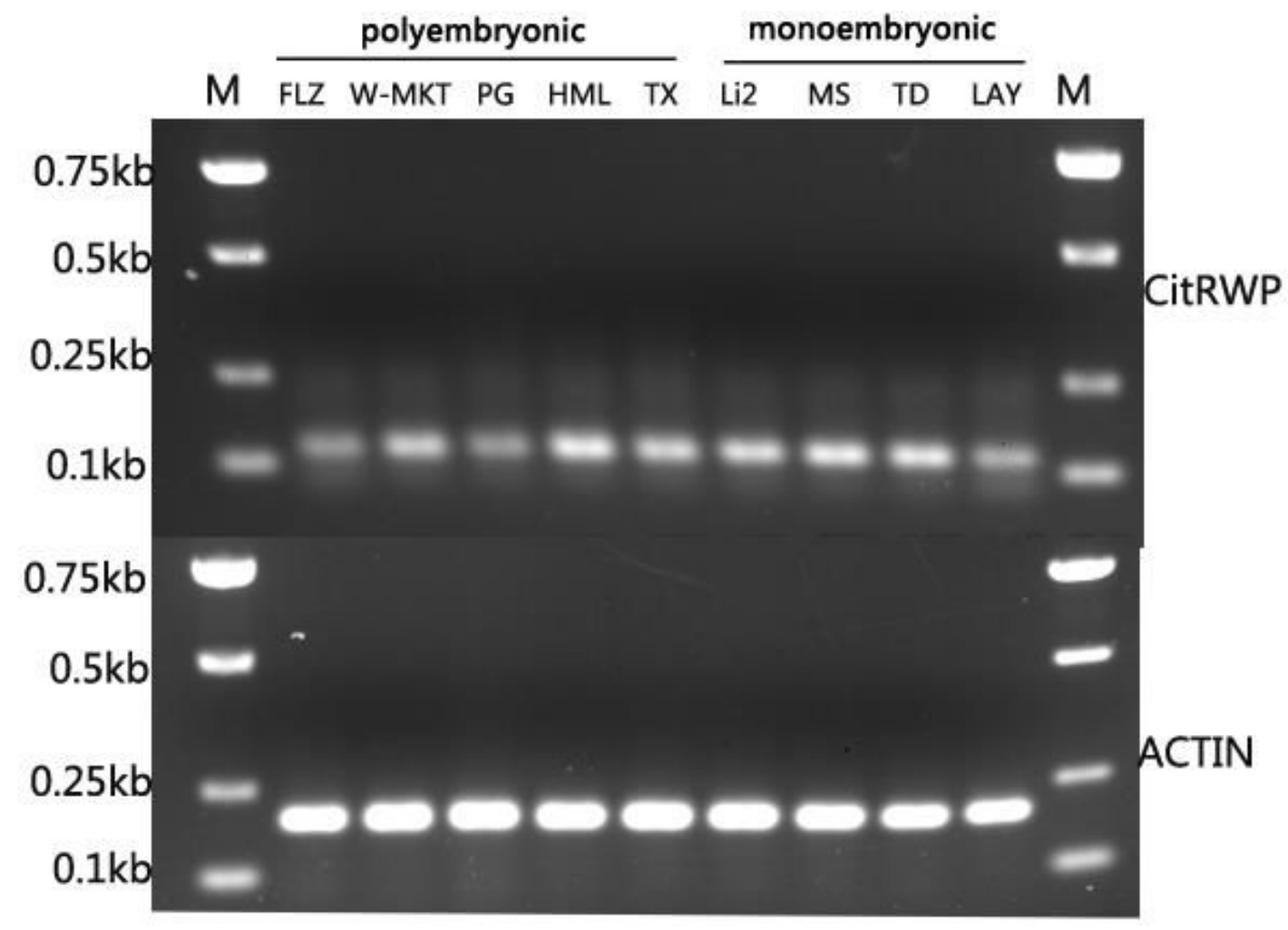

Figure 3

Gene expression pattern of CitRWP in ovule of different embryogenic genotypes by RT-PCR. ACTIN was the reference gene. FLZ: Flying dragon trifoliate orange, W-MKT: W-Murcott tangor, PG: Ponkan, HML: Hamlin sweet orange, TX: Tarocco blood orange, Li2: Licheng No. 2 sweet orange, MS: Masai sweet orange, TD: Tiandan sweet orange, LAY: Longan pummelo. 


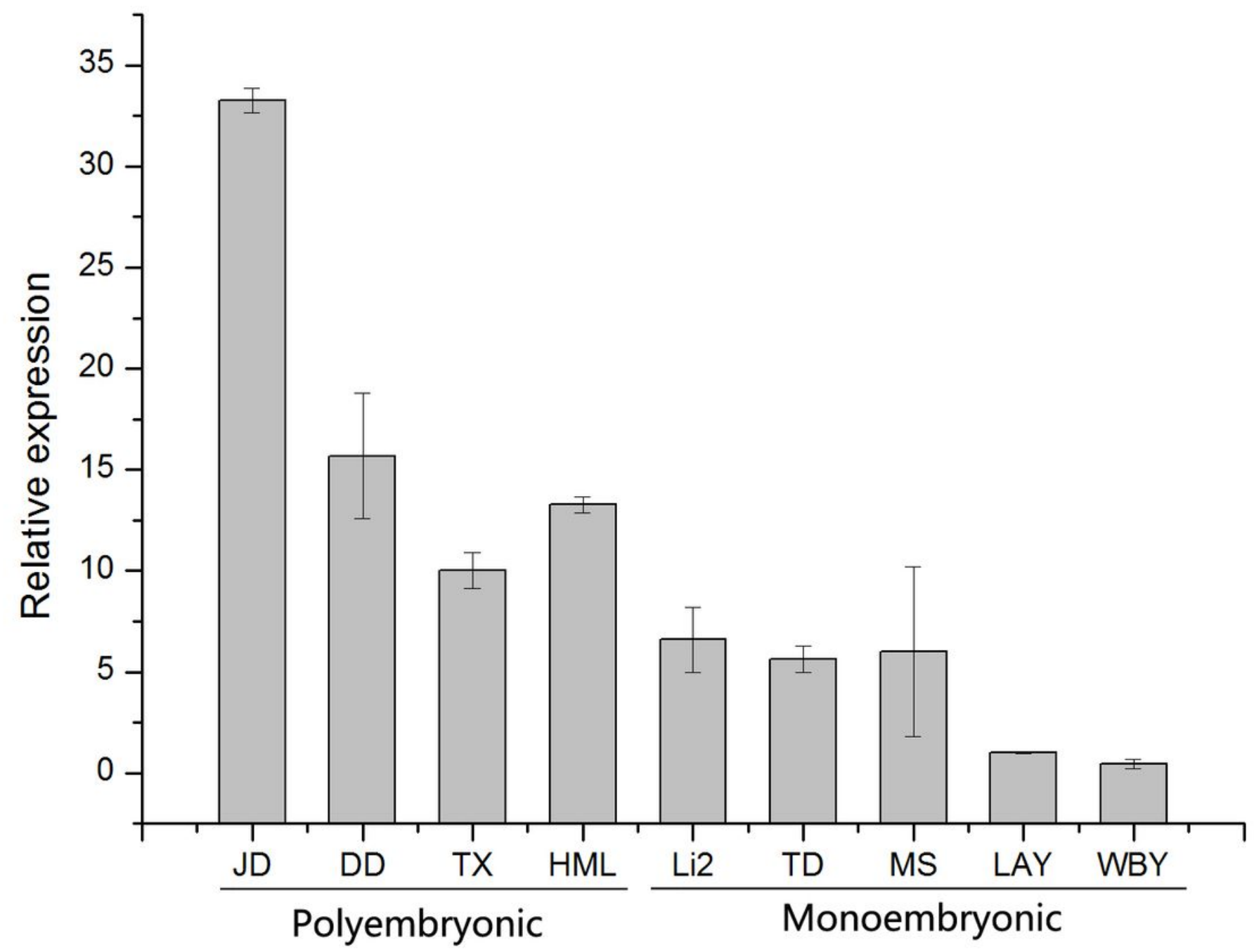

Figure 4

Relative expression of CitRWP in ovule of different embryonic genotypes in 2018. JD: Meiwa kumquat, DD: Daidai, HML: Hamlin sweet orange, TX: Tarocco blood orange, Li2: Licheng No. 2 sweet orange, MS: Masai sweet orange, TD: Tiandan sweet orange, LAY: Longan pummelo, WBY: Banpeiyu pummelo. 


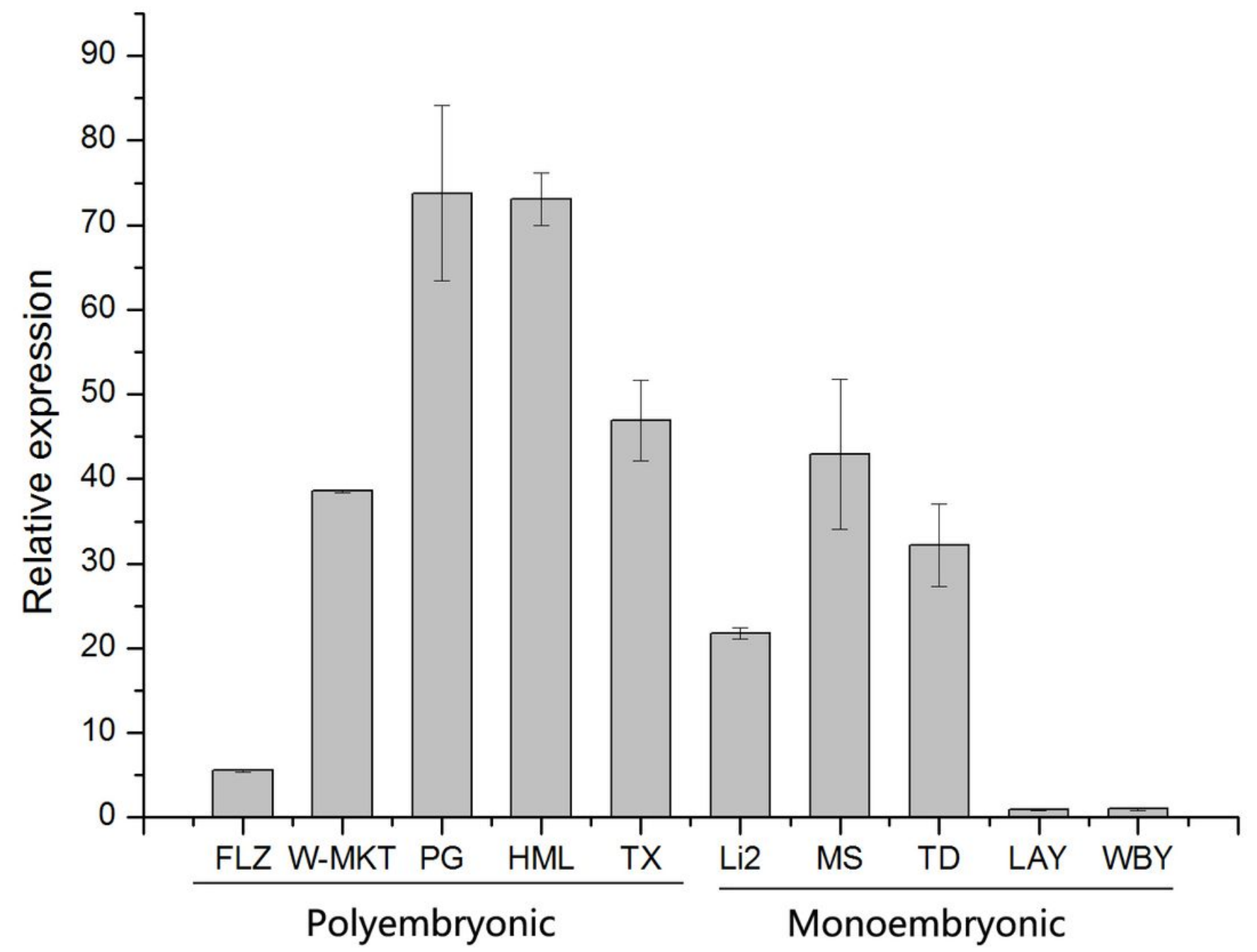

Figure 5

Relative expression of CitRWP in ovule of different embryonic genotypes in 2019. FLZ: Flying dragon trifoliate orange, W-MKT: W-Murcott tangor, PG: Ponkan, HML: Hamlin sweet orange, TX: Tarocco blood orange, Li2: Licheng No. 2 sweet orange, MS: Masai sweet orange, TD: Tiandan sweet orange, LAY: Longan pummelo, WBY: Banpeiyu pummelo. 


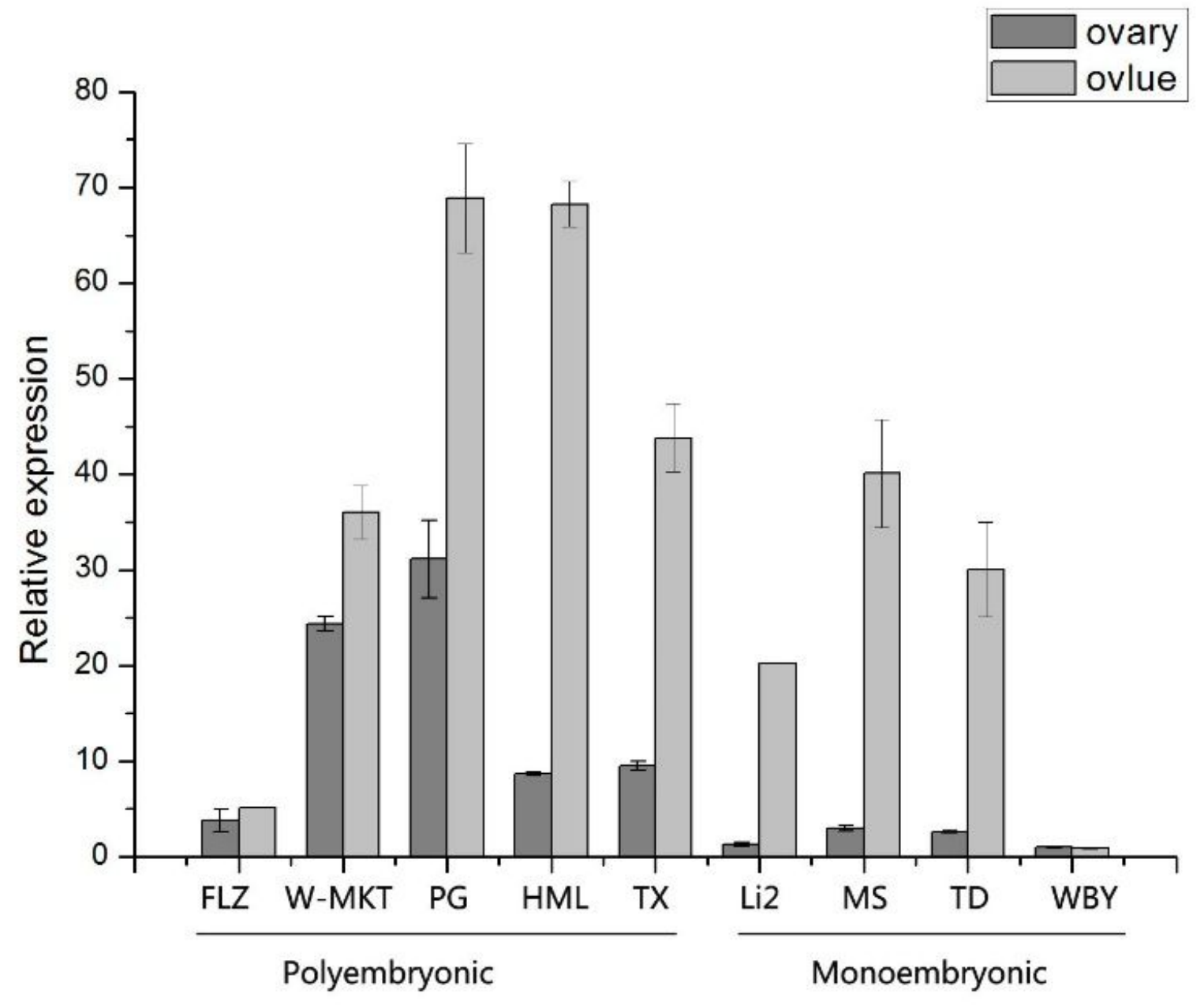

Figure 6

Correlation analysis on CitRWP relative expression of ovule and ovary in citrus. By Pearson correlation analysis, $\mathrm{R}=0.61, \mathrm{P}=0.04 *(<0.05)$ the expression of CitRWP in ovary and ovules was significantly positively correlated at the 0.05 level (unilateral). FLZ: Flying dragon trifoliate orange, W-MKT: W-Murcott tangor, PG: Ponkan, HML: Hamlin sweet orange, TX: Tarocco blood orange, Li2: Licheng No. 2 sweet orange, MS: Masai sweet orange, TD: Tiandan sweet orange, LAY: Longan pummelo, WBY: Banpeiyu pummelo. 


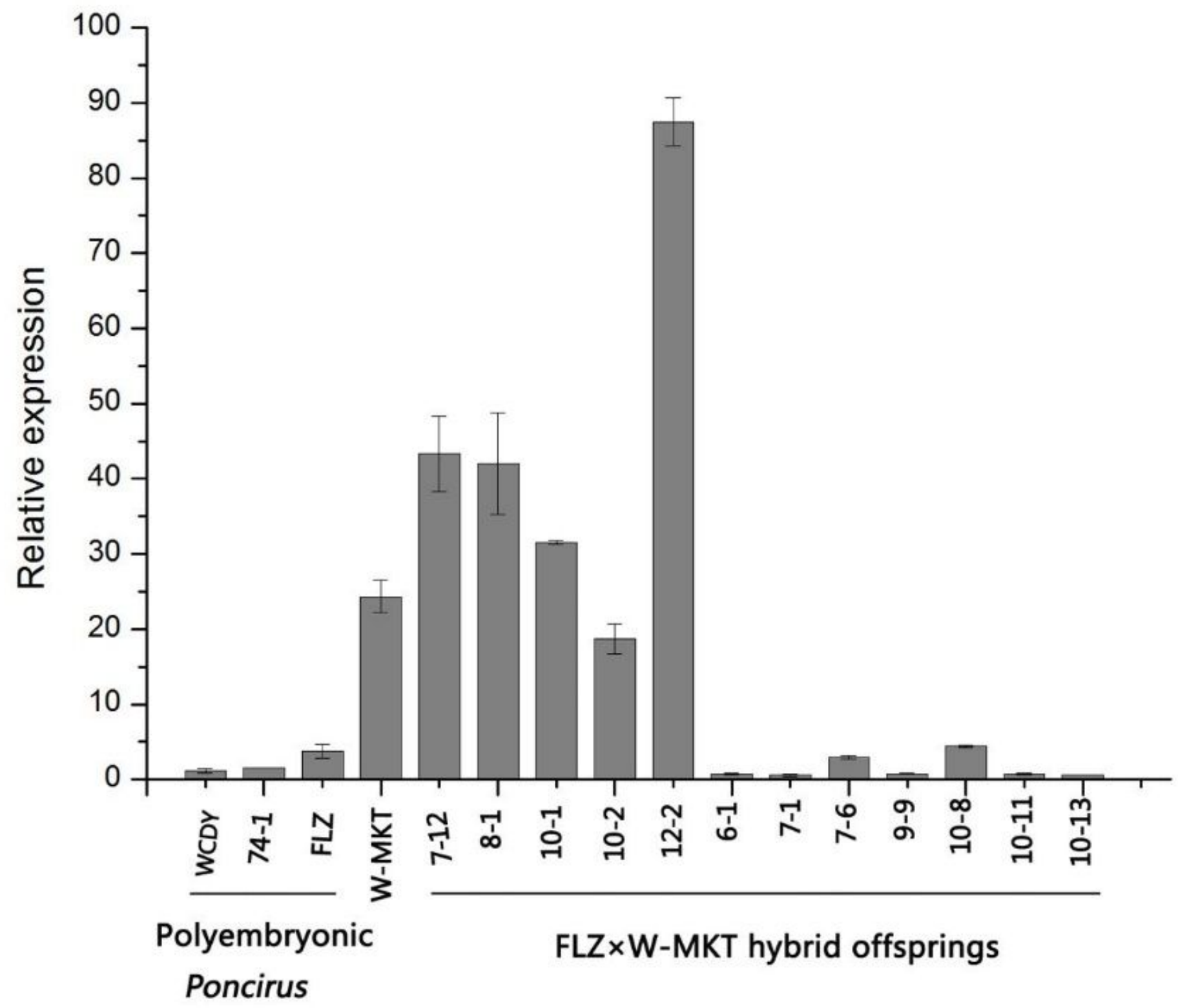

Figure 7

Relative expression of CitRWP in ovary of trifoliate orange genotypes and offsprings of W-Murcott tangor $\times$ Flying dragon trifoliate orange. WCDY: Wangcang daye trifoliate orange, 74-1:74-1 trifoliate orange, FLZ: Flying dragon trifoliate orange区W-MKT: W-Murcott tangor. 


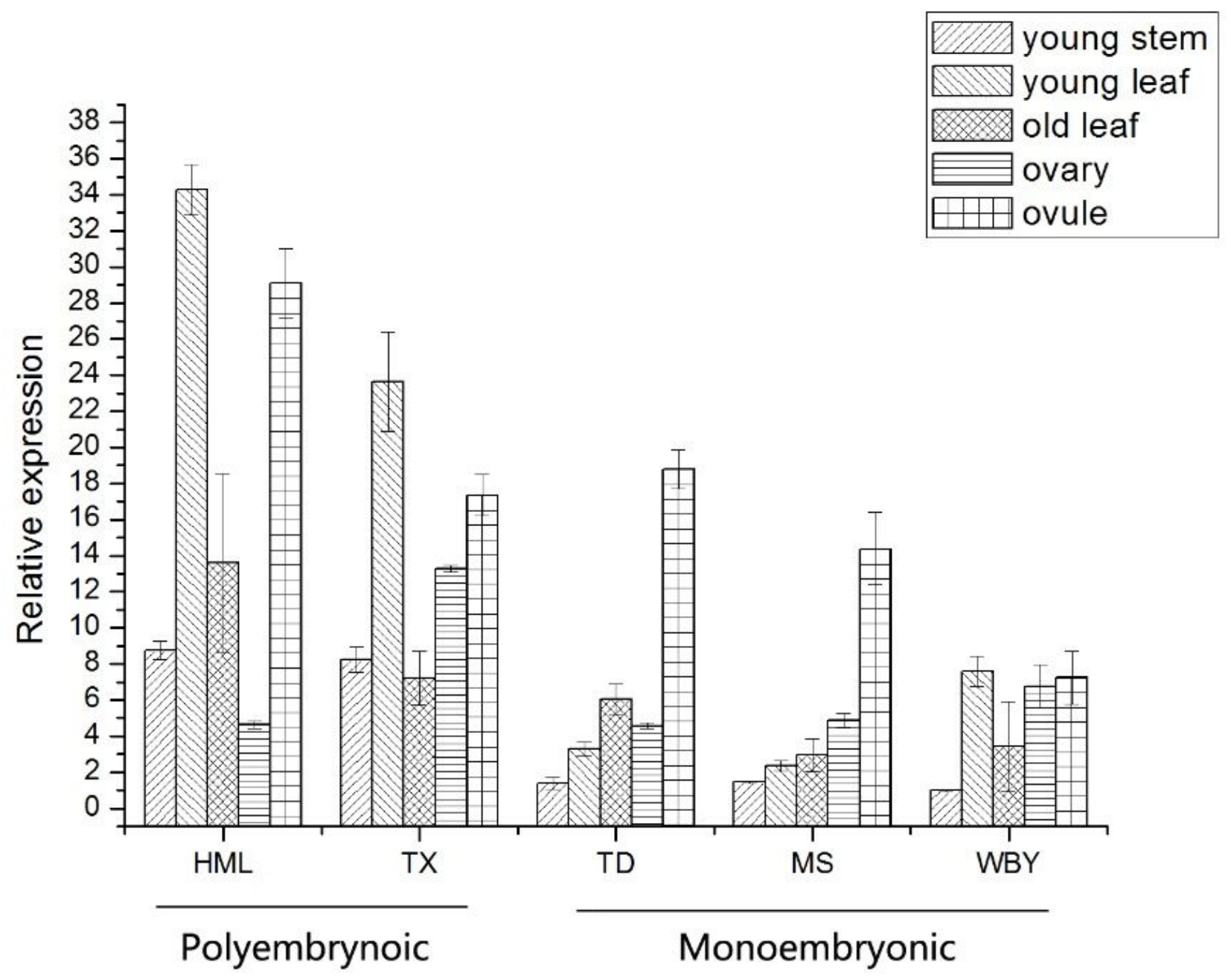

Figure 8

Relative expression of CitRWP in different tissues or organs of different embryonic genotypes. HML: Hamlin sweet orange, TX: Tarocco blood orange, TD: Tiandan sweet orange, MS: Masai sweet orange, WBY: Banpeiyu pummelo. 


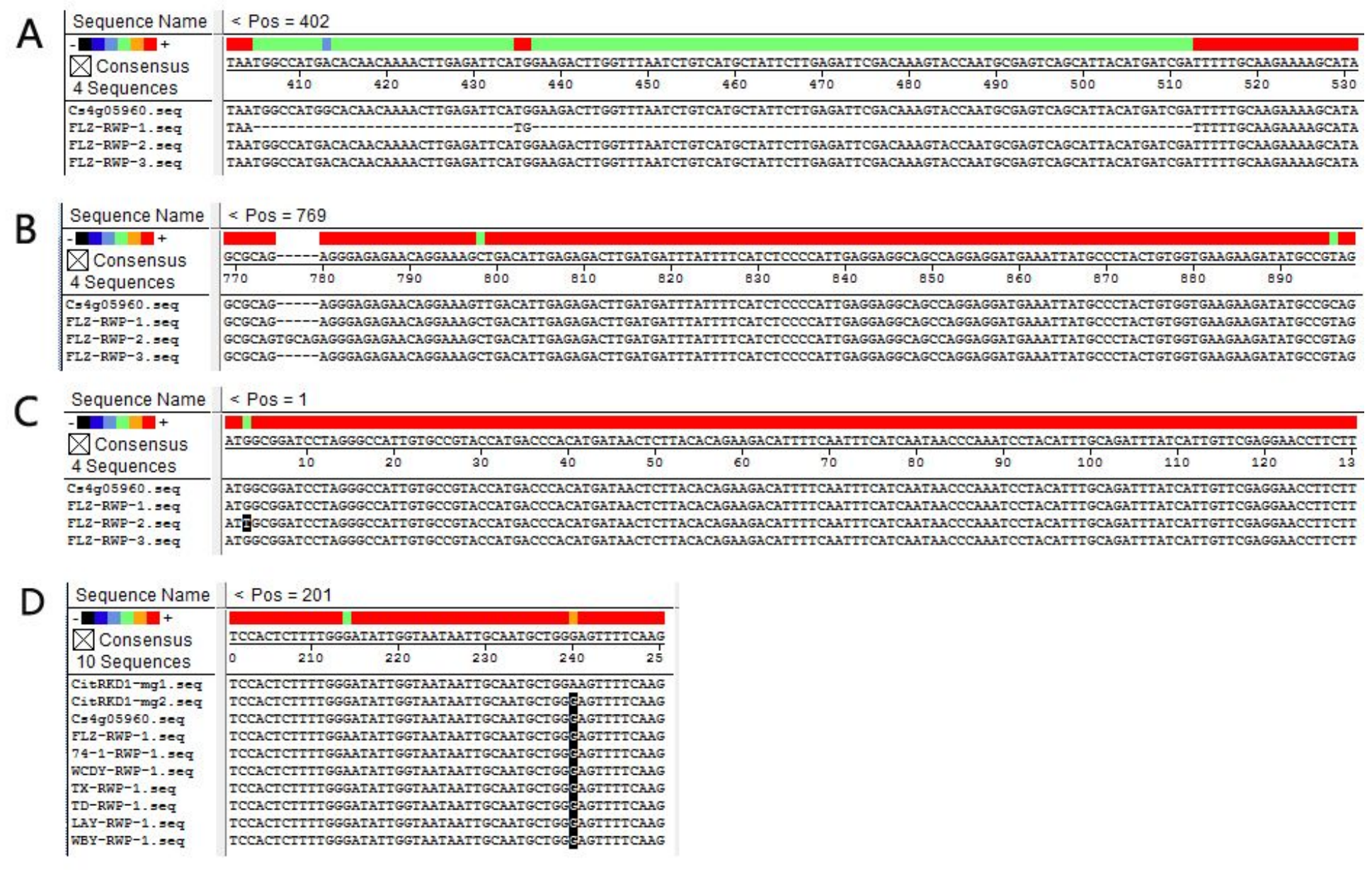

\section{Figure 9}

Variation position in cloned CitRWP cDNA sequences. Cs4g05960: CitRWP CDS sequence in sweet orange [25]. CitRKD1-mg1 and CitRKD1-mg2: CitRWP cDNA sequence in Satsuma mandarin [18]. FLZ-RWP-1: Flying dragon trifoliate orange CitRWP clone, 74-1-RWP-1: 74-1 trifoliate orange CitRWP clone, WCDY-RWP-1: Wangcang daye trifoliate orange CitRWP clone, TX-RWP-1: Tarocco blood orange CitRWP clone区TD-RWP-1: Tiandan sweet orange CitRWP clone, LAY-RWP-1: Longan pummelo CitRWP clone, WBY-RWP-1: Banpeiyu pummelo CitRWP clone. (A) type I variation (deletion of a $106 \mathrm{bp}$ fragment at $406 \mathrm{bp}$ position, relative to the start codon, the same hereinafter), (B) type II variation (insertion of 5bp -TGCAG- at 774bp position), (C) type III variation (mutations in the start-codon or stop-codon). (D) SNP at the 240bp position. All our cloned cDNA sequences were identical with CitRKD1-mg2 (only partial clone sequencing results are shown).

\section{Supplementary Files}

This is a list of supplementary files associated with this preprint. Click to download.

- Additional.docx 